\title{
PFT Regimen
}

National Cancer Institute

\section{Source}

National Cancer Institute. PFT Regimen. NCI Thesaurus. Code C9943.

A chemotherapy regimen consisting of fluorouracil, melphalan, and tamoxifen that may be used in the treatment of breast cancer. 